Revue internationale P.M.E.

Économie et gestion de la petite et moyenne entreprise

\title{
Le développement international des PME : une approche par les réseaux
}

\section{Wafi Chtourou}

Volume 18, numéro 1, 2005

URI : https://id.erudit.org/iderudit/1008471ar

DOI : https://doi.org/10.7202/1008471ar

Aller au sommaire du numéro

\section{Éditeur(s)}

Presses de l’Université du Québec

ISSN

0776-5436 (imprimé)

1918-9699 (numérique)

Découvrir la revue

Citer cette note

Chtourou, W. (2005). Le développement international des PME : une approche par les réseaux. Revue internationale P.M.E., 18(1), 73-102.

https://doi.org/10.7202/1008471ar
Résumé de l'article

Plusieurs approches théoriques sont fournies pour appréhender le développement international des entreprises. Cependant, il apparaît que le cadre conceptuel disponible à ce jour ne permet pas la prise en compte de l'ensemble des spécificités liées aux PME. En effet, les logiques proposées sont pour l'essentiel propres au champ de la grande entreprise. Afin de remédier à cette lacune, nous proposons une réflexion sur l'extension du paradigme éclectique (Dunning, 1977, 1988, 1993, 1995, 2001) qui, comme le soulignent de nombreux auteurs, représente une synthèse des différentes approches. En analysant les réseaux dans une perspective stratégique, ce travail se propose de mettre l'accent sur les avantages procurés par la coopération interentreprises. Afin de répondre à cette problématique, nous présenterons tout d'abord brièvement les fondements théoriques de l'internationalisation des entreprises en général et des PME en particulier, en apportant un soin particulier à la description du modèle issu du paradigme éclectique. Dans un second temps, nous discuterons à la lumière des opportunités offertes par les relations réticulaires et à partir d'un cas concret, les apports de ces derniers aux PME. Enfin, nous tenterons de proposer un cadre-synthèse mieux adapté
Ce document est protégé par la loi sur le droit d'auteur. L'utilisation des services d’Érudit (y compris la reproduction) est assujettie à sa politique d'utilisation que vous pouvez consulter en ligne.

https://apropos.erudit.org/fr/usagers/politique-dutilisation/ 
Notes de recherche

\title{
Le développement international des PME: une approche par les réseaux
}

\author{
Wafi CHTOUROU \\ Institut d'administration des entreprises \\ Université Jean-Moulin Lyon III
}

MOTS CLÉS
Internationalisation - Théorie - PME
Avantages spécifiques à l'entreprise - Avantages liés aux réseaux

\section{RÉSUMÉ}

Plusieurs approches théoriques sont fournies pour appréhender le développement international des entreprises. Cependant, il apparaît que le cadre conceptuel disponible à ce jour ne permet pas la prise en compte de l'ensemble des spécificités liées aux PME. En effet, les logiques proposées sont pour l'essentiel propres au champ de la grande entreprise. Afin de remédier à cette lacune, nous proposons une réflexion sur l'extension du paradigme éclectique (Dunning, 1977, 1988, 1993, 1995, 2001) qui, comme le soulignent de nombreux auteurs, représente une synthèse des différentes approches. En analysant les réseaux dans une perspective stratégique, ce travail se propose de mettre l'accent sur les avantages procurés par la coopération interentreprises. Afin de répondre à cette problématique, nous présenterons tout d'abord brièvement les fondements théoriques de l'internationalisation des entreprises en général et des PME en particulier, en apportant un soin particulier à la description du modèle issu du paradigme éclectique. Dans un second temps, nous discuterons à la lumière des opportunités offertes par les relations réticulaires et à partir d'un cas concret, les apports de ces derniers aux PME. Enfin, nous tenterons de proposer un cadre-synthèse mieux adapté.

\section{L'AUTEUR}

$W_{A F I}$ CHTOUROu est attaché d'enseignement et de recherche à l'Institut d'administration des entreprises de l'Université Jean-Moulin Lyon III. Diplômé de l'ESC Tunis et de l'IAE de Lyon, il poursuit actuellement ses recherches sur le développement international des PME, les enjeux stratégiques de l'information et des connaissances et le partage au sein des réseaux d'entreprises. Adresse: 6, cours Albert-Thomas, B.P. 8242, 69355 Lyon, Cédex 8, France. Téléphone: 4787871 58, télécopieur: 4787877 50. Courriel: <chtourou@univ-lyon3.fr>.

(C) 2005 - Presses de l'Université du Québec

Édifice Le Delta I, 2875, boul. Laurier, bureau 450, Sainte-Foy, Québec G1V 2M2 • Tél.: (418) 657-4399 - www.puq.ca

Tiré de: Revue internationale P.M.E., vol. 18, $\mathrm{n}^{\circ} 1$, sous la direction de Louis Raymond • PME1801N

Tous droits de reproduction, de traduction et d'adaptation réservés 


\begin{abstract}
If many theoretical frameworks have been suggested in order to explain the international developement of the firms, it seems that these theories are unable to take into account small and medium enterprises specificities. In order to fill this theoretical gap, we have decided to use the eclectic theory (Dunning, 1977, 1988, 1993, 1995, 2001). This theory, as pointed out by many researchers, can be conceptualized as a synthesis of the other theories. Analysing the networks through a strategical point of view, this article aims at focusing on the advantages procured by interfirms cooperation. To do this, we will firstly propose some short theoretical bases concerning the internationalisation of the firms. So that, we will study specifically the "eclectic model". We will then focus on SMEs specificities that we will discuss using a network perspective. Finally, starting from a concrete case, which allows to make a confrontation between the main problems SMEs are facing and reticular relations opportunities, we will try to propose a framework that could fit to SMEs specificities.
\end{abstract}

\title{
RESUMEN
}

Si varios campos téoricos han sido propuestos para exponer el desarrollo internacional de las empresas, queda claro que el ámbito conceptual no permite tomar en cuenta todas las especificidades de las PyMEs. Con el fin de clarificar esta problemática, proponemos una reflexión acerca de la teoría ecléctica (Dunning, 1977, 1988, 1993, 1995, 2001), que representa una síntesis de los diferentes estudios, como lo ponen de relieve numerosos autores. Analizando las redes en una perspectiva estratégica, este trabajo se concentra en las ventajas de la cooperación entre las empresas. Con el fin de tratar de esta problemática, presentaremos en primer lugar brevemente los fundamentos teóricos de la internacionalización de las empresas, teniendo cuidado en la presentación del modelo resultado de la teoría ecléctica. En segundo lugar, nos concentraremos sobre las especifidades de las PyMEs que trataremos con la ayuda de un enfoque basado sobre las redes. Para finalizar presentaremos un caso concreto, favoreciendo la confrontaciòn entre las mayores dificultades encontradas por las PyMEs y las oportunidades detectadas por las relaciones reticulares, intentaremos proponer una síntesis teórica más apropiada.

\section{ZUSAMMENFASSUNG}

Mehrere theoretische Konzepte befassen sich mit der Internationalisierung von Unternehmen. Die verwendeten, aktuell verfügbaren, konzeptionellen Rahmenbedingungen eignen sich jedoch nicht, die Gesamtheit der Eigenheiten von KMUs im Internationalisierungsprozess zu berücksichtigen. In der Tat sind die meisten Konzepte in erster Linie für grosse Unternehmen zu gebrauchen. Um diese Forschungslücke zu schliessen, schlagen wir eine erweiterte Betrachtung des eklektischen Paradigmas (Dunning, 1977,1988,1993,1995, 2001) vor, dass, wie von zahlreichen Autoren unterstrichen wird, eine Synthese der verschiedenen Ansätze darstellt. Diese Arbeit legt den Fokus auf die strategischen, zwischenbetrieblichen Partnerschaften und deren Vorteile in der Internationalisierung. Zunächst verschaffen wir uns einen kurzen Überblick über die theoretischen Grundlagen der Internationalisierung von Unternehmen im Allgemeinen und von KMU im Besonderen. Speziellem Wert legen

Revue internationale P.M.E., vol. $18, \mathrm{n}^{\circ} 1,2005$

(C) 2005 - Presses de l'Université du Québec

Édifice Le Delta I, 2875, boul. Laurier, bureau 450, Sainte-Foy, Québec G1V 2M2 • Tél.: (418) 657-4399 - www.puq.ca

Tiré de: Revue internationale P.M.E., vol. 18, $\mathrm{n}^{\circ} 1$, sous la direction de Louis Raymond PME1801N

Tous droits de reproduction, de traduction et d'adaptation réservés 
wir dabei auf die Betrachtung des bereits erwähnten eklektischen Paradigmas. In einer zweiten Phase betrachten wir die Gelegenheiten im Internationalisierungsprozess, die durch Beziehungsnetzwerke entstehen. Anhand eines konkreten Fallbeispieles werden diese Gelegenheiten illustriert. Schließlich versuchen wir, einen besser angepßt Synthese-Rahmen für die beschriebene Problematik anbieten zu können.

\section{Introduction}

La présence des entreprises sur les marchés étrangers a pris depuis plusieurs années une place importante dans la réflexion stratégique des dirigeants. Premières génératrices d'emplois à travers le monde (Julien, 2002), les PME qui saisissent les occasions de développement international en collaborant avec des partenaires locaux ou étrangers deviennent de plus en plus nombreuses. Quel que soit le partenaire, en vue de mettre en commun des ressources, partager les risques et les coûts, atteindre une taille critique, imposer des normes au marché, aboutir à une nouvelle technologie, etc., les nouvelles options stratégiques à dominante relationnelle se présentent comme une démarche recherchée par les entreprises partout à travers le monde. Dans cette optique, décrire les avantages des réseaux de coopération et leurs apports relativement aux caractéristiques propres des entreprises nous semble utile pour mieux comprendre le développement international des PME. L'objectif est de faire valoir que l'action en réseau constitue une perspective flexible et bien adaptée aux PME soucieuses de mener des activités à l'international. L'accent est mis sur l'apport en avantages coopératifs pour ces dernières.

Une brève présentation des différents courants théoriques sur l'internationalisation des firmes précédera notre étude des fondements du paradigme éclectique soumis par Dunning (1977) ainsi que son modèle OLI ${ }^{1}$ (1988, 1993, 1995, 2001, 2002) jugé par plusieurs auteurs comme une synthèse de la recherche contemporaine sur l'internationalisation. Notre choix de focalisation sur le paradigme éclectique se fonde essentiellement sur une double raison: la première rejoint l'objectif de l'auteur qui vise à proposer un modèle qui dépasse la simple conception parcellaire pour une interprétation plus intégrative; la seconde s'inspire d'une volonté d'exploration théorique pour les PME.

À partir d'une analyse de la théorie disponible, nous pointons dans un premier temps la nécessité d'un champ théorique adapté aux PME. Dans un deuxième temps et à la lumière d'une étude de cas en profondeur, nous nous intéressons aux apports de la coopération en réseaux. Enfin, une discussion sur la compatibilité du paradigme éclectique pour la PME alimentera notre réflexion sur la possibilité d'adaptation en utilisant une approche par les réseaux.

1. OLI: Ownership-specific advantages, Localisation advantages and Internalization advantages.

Revue internationale P.M.E., vol. 18, nº 1, 2005

(C) 2005 - Presses de l'Université du Québec

Édifice Le Delta I, 2875, boul. Laurier, bureau 450, Sainte-Foy, Québec G1V 2M2 - Tél.: (418) 657-4399 - www.puq.ca

Tiré de: Revue internationale P.M.E., vol. 18, $\mathrm{n}^{\circ} 1$, sous la direction de Louis Raymond • PME1801N

Tous droits de reproduction, de traduction et d'adaptation réservés 


\section{Le champ théorique disponible}

Comme la littérature est très abondante, nous avons jugé bon de procéder à un recensement avant de nous attarder à l'effort de synthèse le plus pertinent.

Tableau 1

Les principales approches traditionnelles sur l'internationalisation des entreprises

\begin{tabular}{|c|c|c|}
\hline $\begin{array}{l}\text { Approches } \\
\text { théoriques }\end{array}$ & Logiques fournies & Principaux auteurs \\
\hline Pays & $\begin{array}{l}\text { Avantage absolu } \\
\text { Avantages comparatifs } \\
\text { Dotation factorielle des pays }\end{array}$ & $\begin{array}{l}\text { Smith (1776) } \\
\text { Ricardo (1817) } \\
\text { Heckscher (1949) } \\
\text { Ohlin et Heckscher (1933) } \\
\text { Mundell (1957) } \\
\text { Chipman (1965) }\end{array}$ \\
\hline Marché & $\begin{array}{l}\text { Imperfection des marchés } \\
\text { cycle de vie d'un produit } \\
\text { Internalisation des activités } \\
\text { Conditions de la concurrence } \\
\text { Barrières à l'entrée } \\
\text { Coûts de transactions } \\
\text { Spécialisation sur la chaîne } \\
\text { de valeur }\end{array}$ & $\begin{array}{l}\text { Coase (1937) } \\
\text { Byé (1987) } \\
\text { Hymer (1967) } \\
\text { Kindleberger (1969) } \\
\text { Williamson (1975) } \\
\text { Rugman et Lorraine (1985) } \\
\text { Porter (1986) }\end{array}$ \\
\hline Firme & $\begin{array}{l}\text { Organisation de la firme } \\
\text { Structure opérationnelle } \\
\text { Maximisation du profit } \\
\text { et de la valeur }\end{array}$ & $\begin{array}{l}\text { Ferguson (1996) } \\
\text { Cyert et March (1964) } \\
\text { March et Simon (1969) } \\
\text { Buckley et Casson (1976) }\end{array}$ \\
\hline Capital & $\begin{array}{l}\text { Écart de rémunération } \\
\text { du capital } \\
\text { Diversification du portefeuille } \\
\text { Réduction du risque }\end{array}$ & $\begin{array}{l}\text { Kindleberger (1969) } \\
\text { Caves (1982) } \\
\text { Jaquemot (1990) } \\
\text { Björkman (1990) }\end{array}$ \\
\hline $\begin{array}{l}\text { Comportement du } \\
\text { décideur }\end{array}$ & $\begin{array}{l}\text { Rationalité limitée } \\
\text { Démarche prudente } \\
\text { Démarche en étapes } \\
\text { Processus social } \\
\text { Processus progressif } \\
\text { Processus cumulatif } \\
\text { Processus dynamique } \\
\text { (Uppsala) }\end{array}$ & $\begin{array}{l}\text { March et Simon (1969) } \\
\text { Johanson et Weidersheim (1975) } \\
\text { Johanson et Vahlne (1977) } \\
\text { Welch et Luostarinen (1988) } \\
\text { Johanson et Vahlne (1993) } \\
\text { Phatak (1992) } \\
\text { Soerensen et Nedergaard (1993) } \\
\text { Aharoni (1996) } \\
\text { Manolova } \text { et al. (2002) }\end{array}$ \\
\hline
\end{tabular}




\subsection{Les approches traditionnelles sur le développement international}

Les réflexions théoriques sur le développement international des entreprises s'articulent essentiellement autour de cinq axes: le pays, le marché, le capital, la firme et le comportement du décideur ${ }^{2}$. Depuis Smith $(1776)^{3}$ et Ricardo (1817), les théories liées au pays représentent les premières tentatives d'explication du phénomène (Heckscher, 1949; Ohlin et Heckscher, 1933; Samuelson, 1948). Les travaux effectués par Mundell (1957) et Chipman (1965) rejoignent la logique économique de différence de dotation des pays, des avantages offerts et du transfert de capitaux comme substitut au commerce. L'internalisation des activités (Hymer, 1968), l'imperfection des marchés (Kindleberger, 1969), les conditions de la concurrence, le cycle de vie d'un produit (Vernon, 1966) et le contournement des barrières à l'entrée ont souvent alimenté les pensées théoriques liées au marché. Byé (1987) et Williamson (1975) ont constaté à ce niveau la dualité entre le marché et la firme, entre les échanges extérieurs et les flux internes à la firme. Une explication, par l'économie des coûts de transaction (Coase, 1937), défend l'idée selon laquelle une firme internalise ses activités aussi longtemps que les coûts pour externaliser ses transactions sont moins importants que ceux qui seraient requis en recourant au marché. À ce sujet, Williamson (1975) stipule que les agents économiques éprouvent des difficultés à obtenir et traiter l'information de façon totalement fiable et que, dans ce contexte de complexité et d'incertitude liées à une asymétrie d'information entre acteurs économiques, l'internalisation est susceptible de pallier les carences du marché. La recherche d'une minimisation des coûts de transactions serait donc à l'origine de l'internationalisation. Par ailleurs, devant la forte croissance des flux de capitaux en provenance des États-Unis vers l'Europe après la Seconde Guerre mondiale, certains auteurs (dont Caves, 1982) ont tenté d'expliquer cette situation par les écarts de rémunération entre les deux régions. Une extension de la réflexion mène à envisager la minimisation des risques par la diversification du portefeuille (Jacquemot, 1990). En partant d'une hypothèse de maximisation de valeur sur le marché de la firme, la contribution théorique de Ferguson (1996) a été de déduire des conditions de maximisation des profits une structure précise opérationnelle pour le niveau désiré de capital. Il est supposé alors que toute firme possède différents projets d'investissements possibles (que ce soient des projets nationaux ou étrangers) et qu'elle connaît le coût actuel, qu'elle

2. Terminologie utilisée par Simon (1957).

3. En 1776, dans son ouvrage intitulé Recherche sur la nature et les causes de la richesse des nations, Adam Smith défend l'idée du libre-échange. En s'appuyant sur le principe de la spécialisation des nations, il fait du commerce entre les nations une source de richesses et reprend l'exemple de la manufacture d'épingles dans lequel il montre que la productivité du travail augmente avec la division technique du travail.

Revue internationale P.M.E., vol. 18, n 1, 2005

(C) 2005 - Presses de l'Université du Québec

Édifice Le Delta I, 2875, boul. Laurier, bureau 450, Sainte-Foy, Québec G1V 2M2 • Tél.: (418) 657-4399 - www.puq.ca

Tiré de: Revue internationale P.M.E., vol. 18, $\mathrm{n}^{\circ} 1$, sous la direction de Louis Raymond • PME1801N

Tous droits de reproduction, de traduction et d'adaptation réservés 
peut estimer les flux de rendement attendus pour chacun d'eux, un classement de ces projets sera alors effectué en fonction de leur taux de rendement, pour un taux d'intérêt donné sur le marché.

Aux côtés des théories économiques de maximisation du profit, les théories béhavioristes et organisationnelles (March et Simon, 1969) évoquent les principes fondamentaux de rationalité limitée ${ }^{4}$, de satisfaction et d'organisation sociale et politique pour comprendre les processus décisionnels au sein de l'entreprise. Aharoni (1996) intègre l'internationalisation des entreprises dans un processus social en changement et fait remarquer que la connaissance du marché et les préférences du décideur jouent un rôle très important dans ce processus. Le courant comportementaliste, promu par les Scandinaves (Johanson et Wiedersheim, 1975; Johanson et Vahlne, 1977), constitue également une référence dans les tentatives explicatives du développement international des entreprises. Basé sur l'hypothèse de rationalité limitée et d'information imparfaite dans un contexte d'incertitude, le modèle d'Uppsala fournit une explication de l'internationalisation par une approche progressive et séquentielle. Par opposition à un modèle planifié et rationnel, basé sur une réflexion organisée et un ensemble de paramètres maîtrisés, l'idée d'une démarche prudente et progressive d'internationalisation est défendue. Elle présente l'internationalisation comme issue (et donc solution sous contrainte) pour les entreprises, face à l'étroitesse des marchés nationaux.

D'autres approches se basent sur une interaction entre avantages comparatifs et avantages compétitifs (Kogut, 1985; Porter, 1991). Elles défendent l'idée selon laquelle la localisation des différents maillons de sa chaîne de valeur et l'allocation optimale des ressources sur ces maillons encourage l'internationalisation pour l'entreprise. La dynamique de compétition internationale s'expliquerait autant par des différences entre firmes, que par des différences entre pays (Huault, 1998). Ainsi, entre les avantages économiques, les intérêts stratégiques à l'internationalisation et les économies de coûts, la mosaïque théorique sur le sujet ne nous fournit pas une interprétation unique des motivations à l'internationalisation.

Dans l'effort de synthèse poursuivi depuis une trentaine d'années, une démarche mérite d'être examinée. Au-delà de ces limites (aspect managérial), le paradigme éclectique propose une synthèse de traitements combinant les divers avantages (géographiques, technologiques, organisationnels, etc.) pour fonder le choix de l'internationalisation en faisant intervenir le pays, l'industrie et l'entreprise. Nous présentons dans ce qui suit les principes de cette conception afin de discuter dans un second temps sa pertinence pour les PME.

4. Concept de rationalité limitée introduit par Simon (1957) et March et Simon (1958), qui postulent «que le décideur, bien que doté de buts guidant son activité, est limité d'un point de vue cognitif... face à la complexité des problèmes qu'il traite, il recherche un niveau de satisfaction plutôt qu'un optimum ».

Revue internationale P.M.E., vol. 18, nº 1, 2005

(C) 2005 - Presses de l'Université du Québec

Édifice Le Delta I, 2875, boul. Laurier, bureau 450, Sainte-Foy, Québec G1V 2M2 • Tél.: (418) 657-4399 - www.puq.ca

Tiré de: Revue internationale P.M.E., vol. 18, $\mathrm{n}^{\circ}$ 1, sous la direction de Louis Raymond • PME1801N

Tous droits de reproduction, de traduction et d'adaptation réservés 


\subsection{Le paradigme éclectique: une conception synthétique?}

Hirsch (1976), Caves (1982) et Dunning (1977, puis 1988, 1993, 1995, 2001, 2002) sont les auteurs qui sont allés le plus loin dans les tentatives de dépassement des analyses monolithiques de la firme. Cette école vise à offrir «un cadre général grâce auquel il est possible d'identifier et d'évaluer les facteurs influençant, d'une part, l'engagement initial de l'entreprise dans une production étrangère et, d'autre part, l'évolution de cette production» (Dunning, 1988). Ce cadre général est constitué de trois types d'avantages perçus par l'entreprise. Il se rattache à ce qu'il est convenu d'appeler le paradigme «OLI».

\subsubsection{Les avantages spécifiques à l'entreprise: firme, capital}

Pour s'implanter à l'étranger, une entreprise doit disposer d'avantages distinctifs sur la propriété et la gestion des ressources, par rapport aux autres entreprises du pays d'accueil comme à celles du pays d'origine. Ces avantages sont par exemple liés à des facilités particulières d'accès aux ressources financières, à la possession d'une technologie plus avancée, à l'existence d'économies d'échelle sur le plan de la logistique, de la commercialisation ou de l'approvisionnement ou encore à une expérience plus ancienne; on parlera dans ce cas de «courbe d'apprentissage» privilégiée. Ces avantages spécifiques doivent être exclusifs pendant une période suffisamment longue permettant l'intégration facile et durable de l'entreprise à l'étranger. Il est à noter que certaines caractéristiques du marché local (ressources naturelles, taille du marché national, qualification de la main-d'œuvre, niveau de développement de la technologie, etc.) peuvents'encastrer dans ce type d'avantages.

\subsubsection{Les avantages comparatifs de localisation géographique: pays}

Une entreprise décidera d'exporter sa production d'un pays A vers un pays B si les combinaisons de facteurs sont plus favorables en B qu'en A (Ohlin et Heckscher, 1933, puis Samuelson, 1948). Les analyses du commerce international à l'échelle d'un pays peuvent révéler la présence d'un ensemble d'éléments qui interviennent dans la formation du prix d'un bien donné. Ainsi, on peut distinguer à ce niveau les éléments associés au coût et à la disponibilité des facteurs de production, les éléments liés aux particularités du système institutionnel qui prévaut dans le pays d'implantation (régime fiscal, réglementation du travail, restriction aux échanges, etc.) et les atouts spécifiques de location (qualité du site, proximité des matières premières ou des débouchés, etc.).

Revue internationale P.M.E., vol. 18, $\mathrm{n}^{\circ}$ 1, 2005

(C) 2005 - Presses de l'Université du Québec

Édifice Le Delta I, 2875, boul. Laurier, bureau 450, Sainte-Foy, Québec G1V 2M2 • Tél.: (418) 657-4399 - www.puq.ca

Tiré de: Revue internationale P.M.E., vol. 18, $\mathrm{n}^{\circ} 1$, sous la direction de Louis Raymond • PME1801N

Tous droits de reproduction, de traduction et d'adaptation réservés 


\subsubsection{Les avantages de l'internalisation: marché}

Une entreprise désirant s'intégrer à l'étranger peut détenir un avantage d'un autre ordre: celui qui consiste à ne plus transiter par le marché pour conquérir des places dans le pays d'accueil. La globalisation des activités permet de compenser les imperfections observées sur le marché des facteurs, de produits et de biens, ainsi que de déduire les coûts de transaction des marchés. La firme choisira donc l'investissement direct - et donc la création d'un espace mieux intégré - si elle peut tirer de l'exploitation directe de cet avantage (associé à un mode d'organisation «internalisé») un profit plus important que si elle décidait simplement de vendre ses produits ou de louer ses compétences (brevets, licences, etc.) à un concessionnaire d'un pays étranger.

La détention combinée de ces trois types d'avantages confère à une firme à un moment donné et sur des marchés déterminés, des avantages globaux à l'internationalisation. Ainsi, elle pourra opter pour un développement à l'étranger à travers l'investissement direct. La présence de un ou deux types d'avantages parmi les trois peut permettre à l'entreprise d'opter pour une simple exportation ou pour un accord de partenariat avec tiers étranger. Ce raisonnement n'est pas exclusif, d'ailleurs comme le dit l'auteur lui-même, «une seule explication ne peut rendre compte de toutes les formes d'internationalisation» (Dunning, 1995). C'est dans cette optique que nous nous intéressons dans ce qui suit à l'internationalisation des PME.

\subsection{Internationalisation des PME: la nécessité d'un champ théorique adapté}

Par une distinction entreprise exportatrice/non exportatrice, Cavusgil (1976) avait conclu que l'impact de taille des firmes sur le développement international est significatif dans les cas où ces dernières sont très petites. Plus tard, l'auteur stipule dans un article rédigé avec Nevin (Cavusgil et Nevin, 1981) que la probabilité d'être exportateur augmente avec la taille de l'entreprise et que le volume des ventes est un déterminant significatif dans le comportement à l'exportation. Piore et Sabel (1984) et Reid (1984) se sont intéressés à l'impact de la taille sur le comportement à l'exportation des PME italiennes. La première idée défendue recommande aux PME de se développer sur le marché local et d'éviter une activité risquée comme l'exportation. En revanche, les grandes entreprises doivent exporter pour soutenir la croissance de leurs ventes. Aaby et Slater (1989) stipulent à cet égard que les grandes compagnies ont des avantages - taille connexe - qui leur permettent de s'engager plus efficacement dans l'exportation. L'exception des petites entreprises spécialisées ou maîtrisant une technologie de pointe a été bien considérée, dans la mesure où le manque de ressources n'est plus considéré comme une contrainte.

Revue internationale P.M.E., vol. 18, $n^{\circ}$ 1, 2005

(C) 2005 - Presses de l'Université du Québec

Édifice Le Delta I, 2875, boul. Laurier, bureau 450, Sainte-Foy, Québec G1V 2M2 • Tél.: (418) 657-4399 - www.puq.ca

Tiré de: Revue internationale P.M.E., vol. 18, $\mathrm{n}^{\circ} 1$, sous la direction de Louis Raymond $\cdot$ PME1801N

Tous droits de reproduction, de traduction et d'adaptation réservés 
Aux côtés des travaux sur le comportement à l'exportation, les tentatives sur le processus d'internationalisation des PME se sont souvent limitées à l'exportation comme modalité et ont souvent trouvé leur origine dans les explications comportementalistes d'incertitude et d'action prudente et progressive (Johanson et Vahlne, 1977). La revue critique de Leonidou et Katsikeas (1996) retrace les principales tentatives sur le sujet. Après une revue des différents essais, les auteurs proposent une récapitulation en trois grandes phases: une phase de préengagement de l'entreprise dans des activités d'exportation, une phase initiale d'engagement de l'entreprise dans des activités d'exportation et une dernière phase d'engagement avancé de l'entreprise dans des activités d'exportation.

En mettant l'accent sur le rôle du facteur humain (Aaby et Slater, 1989), Manolova et al. (2002) évoquent l'importance du savoir-faire des dirigeants ainsi que de la perception de ces derniers de leur environnement international. Au-delà de la complexité liée au concept (Julien, 1994; Torrès, 1998), les recherches antérieures sur l'internationalisation ont montré plusieurs différences d'attitudes entre les PME et les grandes entreprises (Cavusgil et Nevin, 1981; Marchesnay, 1991; Leonidou, 1997; Julien, 1998). En effet, ces dernières fournissent en général plus d'énergie côté commercial et orientent leur stratégie marketing de façon plus spécifique vers les besoins des clients internationaux (Bloodgood, Sapienza et Almeida, 1996). D'un autre côté, beaucoup de petites entreprises semblent agir de façon réactive dans leurs opérations internationales (Kaynak, 1992). Démunies le plus souvent d'expériences sur les marchés étrangers, elles se heurtent à la difficulté de juger leurs capacités compétitives et semblent avoir peine à bien orienter leurs stratégies d'exportation (De Toni et Nassimbeni, 2001). Côté financier, les PME aux ressources généralement limitées tendent à éprouver plus de problèmes que les grandes entreprises dans la conquête des marchés étrangers. De plus, les ressources limitées des PME rendent la veille sur la qualité des produits et l'organisation de la distribution de façon appropriée très contrariée (Bilkey et Tesar, 1977); ce qui peut constituer «une masse critique» capable de bloquer cette catégorie d'entreprises.

En dépit des recherches considérables sur le sujet, le développement international des PME n'est que partiellement appréhendé par des logiques et incitations propres aux grandes entreprises. Aucune réponse spécifique aux PME n'est disponible à ce jour. L'hétérogénéité de ces dernières (Torrès, 1999), leur nature multidimensionnelle ainsi que la complexité et la dynamique du phénomène rendent difficile la conceptualisation. En tentant un rapprochement de la théorie existante aux spécificités de la PME, nous allons revenir au modèle du paradigme éclectique dans un essai de réflexion théorique. Pour ce faire, une analyse sur les apports des réseaux est avancée et confortée par l'empirique dans la partie qui suit.

Revue internationale P.M.E., vol. 18, n 1, 2005

(C) 2005 - Presses de l'Université du Québec

Édifice Le Delta I, 2875, boul. Laurier, bureau 450, Sainte-Foy, Québec G1V 2M2 - Tél.: (418) 657-4399 - www.puq.ca

Tiré de: Revue internationale P.M.E., vol. 18, $\mathrm{n}^{\circ} 1$, sous la direction de Louis Raymond PME1801N

Tous droits de reproduction, de traduction et d'adaptation réservés 


\section{Quels apports des réseaux?}

\subsection{Les réseaux: quels apports à l'internationalisation?}

Partant de l'idée selon laquelle il est important d'intégrer les réseaux dans les problématiques de l'avantage concurrentiel, les travaux sur le sujet en management stratégique se sont multipliés ces dernières années (Jarillo, 1988; Nohria et Eccles, 1992; Gulati, Nohria et Zaheer, 2000; Coeurderoy et Ingham, 2004). L'approche structurelle ${ }^{5}$, fondée sur l'analyse concurrentielle (Porter, 1986) ne prévoyait pas de logiques d'échanges autres que les ajustements comportementaux en fonction de la rivalité des forces concurrentielles sur le marché. En soulignant l'intérêt du concept pour une utilisation stratégique, Jarillo (1988) se base sur les études antérieures de Johanson et Mattson (1987) pour établir un rapprochement avec la chaîne de valeur de Porter (1986). Un intérêt particulier étant accordé aux problèmes de conceptualisation des réseaux, l'auteur a choisi de faire des réseaux un outil pour obtenir des avantages concurrentiels et non plus une «métaphore» pour décrire les transformations économiques. Ainsi, il a défini le réseau comme un mode d'organisation particulier, utile aux entrepreneurs dans leur stratégie de positionnement en vue de l'obtention d'un avantage compétitif. D'où le concept utilisé de réseau stratégique, dans la mesure où il se matérialise par «des accords voulus de long terme, entre organisations distinctes. Les firmes ainsi reliées retirent un avantage concurrentiel vis-à-vis des firmes hors réseau». L'auteur recommande donc à la firme de se spécialiser dans des activités de chaîne de valeurs où elle trouvera un avantage concurrentiel. Toutefois, il pointe le fait que l'origine et la base de la définition du réseau se situent hors du champ stratégique et intègrent plus les sciences économiques et sociales, la théorie des organisations et la psychosociologie.

Les travaux en marketing industriel, entrepris par Håkansson (1982) se sont intéressés à l'étude des relations dyadiques entre clients et fournisseurs. L'interaction entre ces derniers peut être investie selon quatre niveaux d'analyse: un processus en interaction dynamique, des relations complexes orientées moyen long terme entre les acteurs, une atmosphère agissant sur l'interaction et, enfin, un environnement général dans lequel s'inscrivent les relations. Il est noté, à ce sujet, que l'atmosphère qui règne autour de la relation est affectée par le système social dans lequel s'inscrit l'interaction. Plus tard, Håkansson et Johanson (1993) ont proposé une réflexion sur la structure des réseaux la posant comme une interaction entre acteurs qui mobilisent des ressources dans le but de réaliser des activités. Ainsi, les acteurs doivent 1) définir les activités à réaliser, 2) identifier les ressources à mobiliser, 3) développer des relations personnelles avec d'autres acteurs, 4) établir

5. «Structure - Comportement - Performance » (SCP), une approche selon laquelle les décisions stratégiques sont affectées par la structure du secteur entre concurrence et monopole.

Revue internationale P.M.E., vol. 18, $n^{\circ}$ 1, 2005

(C) 2005 - Presses de l'Université du Québec

Édifice Le Delta I, 2875, boul. Laurier, bureau 450, Sainte-Foy, Québec G1V 2M2 • Tél.: (418) 657-4399 - www.puq.ca

Tiré de: Revue internationale P.M.E., vol. 18, $\mathrm{n}^{\circ} 1$, sous la direction de Louis Raymond $\cdot$ PME1801N

Tous droits de reproduction, de traduction et d'adaptation réservés 
des relations d'échange qui s'encastrent dans les relations personnelles, 5) accéder aux ressources par l'échange, 6) contrôler ces ressources pour, enfin, 7) contrôler les activités prédéfinies. Toutefois, les auteurs n'indiquent pas de façon précise ce qu'ils entendent par ressources «[...] on parle d'activité quand plusieurs acteurs combinent, développent, échangent ou créent des ressources en utilisant d'autres ressources».

Selon Lecoq (2003), deux grandes tendances peuvent être observées par les auteurs actuels traitant les réseaux d'entreprises. La première considère le réseau interorganisationnel comme une construction volontaire. On y distingue un courant socioéconomique fortement marqué par la théorie des coûts de transaction et une approche en termes de relations stratégie-structure dont les concepts ne sont pas si éloignés du courant précédent. La deuxième tendance analyse l'entreprise dans un réseau social et suppose l'existence de relations avant la création d'un réseau. On y distingue là encore les développements sur «l'embeddedness» (Granovetter, 1985), dont les travaux sont très critiques à l'égard du paradigme transactionnel et les travaux issus du marketing industriel. En effet, l'approche sociale porte un intérêt particulier aux notions liées à la structure, aux liens, au fonctionnement et à l'échange dans les réseaux (Emerson, 1962; Powell, 1990; Uzzi, 1997). Différents types d'échanges informels sont possibles en intégrant la dimension sociale dans les réseaux (information, confiance, amitié, etc.). Ainsi, le processus qui conduit à la coopération interentreprises s'insère donc dans une dynamique sociale qui lui sert de fondement.

Par ailleurs, l'approche évolutionniste s'inscrit dans le même champ théorique que l'approche transactionnelle. D' ailleurs, certains auteurs conviennent de la complémentarité des deux courants (Brousseau, 1996; Defeuilley et Vahabi, 1996). En plaçant la question des compétences au centre de ses constructions (Coriat et Weinstein, 1995), la théorie considère la logique de coopération interorganisationnelle comme réductrice de l'incertitude par ses structures de coordination. La contribution de la théorie évolutionniste dans l'étude des relations interfirmes nous éclaire sur le fait que les activités respectivement similaires, complémentaires et dissemblables doivent faire l'objet d'une coordination au sein de la même entité, par la coopération ou par le marché. Ainsi, les relations entre firmes sont considérées comme des réunions de compétences, dans lesquelles les ressources se combinent naturellement. Cependant, la théorie n'apporte pas d'éclairage sur les modalités de coordination à adopter, nous fournissant surtout une explication cognitiviste des raisons pour lesquelles des actifs peuvent et doivent être gérés en synergie (Brousseau, 1996).

Ainsi, la logique stratégique accorde plus d'attention aux finalités de la relation coopérative (le réseau instrument de mobilisation de ressources), alors que la logique sociale s'intéresse essentiellement à l'échange dans la relation entre

Revue internationale P.M.E., vol. 18, $\mathrm{n}^{\circ} 1,2005$

(C) 2005 - Presses de l'Université du Québec

Édifice Le Delta I, 2875, boul. Laurier, bureau 450, Sainte-Foy, Québec G1V 2M2 - Tél.: (418) 657-4399 - www.puq.ca

Tiré de: Revue internationale P.M.E., vol. 18, $\mathrm{n}^{\circ} 1$, sous la direction de Louis Raymond • PME1801N

Tous droits de reproduction, de traduction et d'adaptation réservés 
acteurs (le réseau support d'intermédiation). Cela nous place devant deux logiques différentes, mais que nous pensons complémentaires pour une meilleure étude du phénomène. Le réseau reflète une situation quelquefois floue, qui s'inscrit dans la logique du développement tant de la stratégie des firmes que dans celle de leur analyse et on envisagera le réseau comme une conséquence des transformations majeures des systèmes économiques (contexte), des modes d'organisation (en interne) et des réactions stratégiques des firmes (relation interne-externe).

\subsection{Présentation du cas: spécificités et contexte de la PME en question}

Ayant choisi de comprendre «comment» la coopération en réseau favorise le développement international des PME grâce aux avantages qu'elle offre, nous avons choisi de procéder à une démarche de recherche longitudinale basée sur la construction de cas (Yin, 1990; De la Ville, 2000). Pour ce faire, nous avons opté pour la multiplicité des sources de données. Trois méthodes de recueil ont été choisies: l'observation, l'analyse de documents (10) et des entretiens semi-directifs (12). Le cas que nous avons choisi de traiter a été construit progressivement ${ }^{6}$ par des sociétés dont nous avons jugé l'apport intéressant pour la société «A». La revue de littérature a guidé nos orientations et choix pratiques, compte tenu du caractère abductif de notre recherche, une longue phase d'observation et d'analyse de documents a précédé l'analyse. Pour des raisons de confidentialité, les noms des sociétés ne sont pas diffusés.

«A» est spécialisée dans la fabrication des profilés en acier. Depuis sa création, la société n'a cessé d'enrichir sa gamme de produits destinée entre autres aux secteurs: agricoles, de transport, du BTP et des fabricants de meubles métalliques. Cette gamme permet actuellement à A de couvrir $92 \%$ des besoins de sa clientèle locale $^{7}$. Bien que difficile à estimer, ce pourcentage est beaucoup plus faible au plan international. Par ailleurs, même si la société cherche toujours à élargir sa gamme et à diversifier ses débouchés, ses produits sont caractérisés par une faible valeur ajoutée de production ${ }^{8}$, ce qui constitue pour la société un véritable obstacle au développement international vu l'importance des coûts. Ne nécessitant pas un apport technologique important, les produits de «A» répondent aux normes de qualité exigée par la clientèle internationale et la société est certifiée ISO 9002. Dans une étude comparative basée sur des documents de comptabilité analytique,

6. Sur une période de six mois.

7. Chiffre issu d'une étude réalisée en janvier 2002, qui pointe une saturation du marché national.

8. Remarque du président-directeur général et du directeur technique de l'entreprise. L’information nous a été communiquée lors des entretiens.

Revue internationale P.M.E., vol. 18, nº 1, 2005

(C) 2005 - Presses de l'Université du Québec

Édifice Le Delta I, 2875, boul. Laurier, bureau 450, Sainte-Foy, Québec G1V 2M2 • Tél.: (418) 657-4399 - www.puq.ca

Tiré de: Revue internationale P.M.E., vol. 18, $\mathrm{n}^{\circ}$ 1, sous la direction de Louis Raymond • PME1801N

Tous droits de reproduction, de traduction et d'adaptation réservés 
Tableau 2

Les matériaux empiriques mobilisés pour la recherche

\begin{tabular}{|c|c|c|c|c|c|c|}
\hline Société & $\begin{array}{l}\text { Raison } \\
\text { sociale }\end{array}$ & $\begin{array}{l}\text { Date de } \\
\text { création }\end{array}$ & Effectif & Activité & Localisation & $\begin{array}{l}\text { Détails } \\
\text { Entretiens* }\end{array}$ \\
\hline A & SA & 1979 & 98 & $\begin{array}{l}\text { Fabrication de tubes } \\
\text { ouverts et soudés } \\
\text { en acier. }\end{array}$ & Tunisie & $\begin{array}{l}\text { PDG: } 3 \times 1 \mathrm{~h} \\
\text { DT: } 1 \times 1 \mathrm{~h} \\
\text { DAF: } 2 \times 1 \mathrm{~h} \\
\text { DC: } 1 \times 1 \mathrm{~h}\end{array}$ \\
\hline B & SARL & 1982 & 12 & $\begin{array}{l}\text { Société de commerce } \\
\text { international } \\
\text { spécialisée dans } \\
\text { l'industrie } \\
\text { mécanique. }\end{array}$ & France & $\mathrm{G}: 2 \times 1 \mathrm{~h}$ \\
\hline $\mathrm{C}$ & SA & 1994 & 38 & $\begin{array}{l}\text { Étude et fabrication } \\
\text { des composants } \\
\text { automobiles et pièces } \\
\text { de rechange. }\end{array}$ & Tunisie & DT: $1 \times 1 \mathrm{~h}$ \\
\hline $\mathrm{D}$ & SA & 1985 & 250 & $\begin{array}{l}\text { Fabrication } \\
\text { d'échafaudages, } \\
\text { matériel pour le } \\
\text { bâtiment et les } \\
\text { collectivités. }\end{array}$ & France & $\begin{array}{l}\text { DT: } 1 \times 1 \mathrm{~h} \\
\text { G, Site TN: } 1 \times 1 \mathrm{~h}\end{array}$ \\
\hline $\mathrm{E}^{* *}$ & SA & 1999 & 50 & $\begin{array}{l}\text { Produits de } \\
\text { collectivité et } \\
\text { barrière de tribunes. }\end{array}$ & Tunisie & D: $1 \times 1 h 30$ \\
\hline
\end{tabular}

nous avons pu remarquer que les coûts du personnel assumés par «A» sont sept fois inférieurs aux coûts européens dans le même secteur. Par ailleurs, les partenaires français et allemands de «A» pensent que le niveau de qualification du personnel tunisien est encore plus bas que celui de l'Europe, mais tend à s'améliorer rapidement; ce qui constitue un avantage pour la société par rapport à ses concurrents étrangers. Aussi, contrairement à plusieurs PME, le taux d'encadrement chez «A» représente un atout non négligeable. En effet, le taux de cadres, d'agents de maîtrise et de techniciens supérieurs par rapport à l'effectif total de l'entreprise est passé de $20 \%$ en 1998 à $34 \%$ en 2002. D'une moyenne d'âge relativement faible (32 ans), ce personnel jouit d'une bonne expérience (tous les cadres et agents de maîtrise ont plus de cinq ans d'ancienneté). Cependant, le diagnostic d'un cabinet externe en ressources humaines relève l'absence de comité de direction impliquant l'ensemble des cadres dans le processus d'amélioration. De plus, l'organisation s'approche du type taylorien, privilégiant ainsi les activités de contrôle des tâches sur les réflexions collectives entre cadres et non-cadres. La gestion des ressources humaines est plutôt axée sur l'administration du personnel.

Revue internationale P.M.E., vol. 18, $\mathrm{n}^{\circ} 1,2005$

(C) 2005 - Presses de l'Université du Québec

Édifice Le Delta I, 2875, boul. Laurier, bureau 450, Sainte-Foy, Québec G1V 2M2 • Tél.: (418) 657-4399 - www.puq.ca

Tiré de: Revue internationale P.M.E., vol. 18, $\mathrm{n}^{\circ} 1$, sous la direction de Louis Raymond • PME1801N

Tous droits de reproduction, de traduction et d'adaptation réservés 
Aidé par le rapport d'un cabinet spécialisé en management stratégique ${ }^{9}$, par l'analyse des entretiens que nous avons effectués et par l'observation, nous avons pu détecter un ensemble de forces et de faiblesses propres à «A», résumées dans le tableau suivant.

Tableau 3

Diagnostic des spécificités de «A»

Forces
Bonne connaissance du dirigeant
des enjeux à l'échelle internationale.
Expérience pratique du marché
international, sur plusieurs pays,
permettant de mieux mesurer
les atouts de A sur chaque marché.
Notoriété reconnue à l'échelle national
et internationale.
Intégration d'une vision stratégique
à moyen terme.
Taille moyenne de la société, autorisant
un positionnement dans une niche de
marché à plus forte valeur ajoutée.
Faiblesses

Absence d'un comité de direction impliquant l'ensemble des cadres dans le processus d'amélioration.

Produits de base à très faible valeur ajoutée.

Outil de production inadapté pour répondre aux exigences quantitatives du marché mondial.

Absence d'une fonction marketing et développement permettant l'identification de débouchés potentiels des tubes spécifiques des clients.

Structure commerciale se basant exclusivement sur des représentants, ce qui limite l'identification des clients potentiels intéressants pour «A».

Coûts de transport réduisant les avantages compétitifs à l'international.

Bonne communication interne.

Situation financière ne permettant pas d'assurer le minimum pour le développement international de la société*.

* Le président-directeur général de la société affirme que, sans la subvention accordée par CEPEX depuis 1995, aucune opération d'exportation n'aurait pu avoir lieu. D'ailleurs, avant cette date, le chiffre d'affaires à l'exportation était nul.

9. ALGOE Management (France).

Revue internationale P.M.E., vol. $18, \mathrm{n}^{\circ} 1,2005$

(C) 2005 - Presses de l'Université du Québec

Édifice Le Delta I, 2875, boul. Laurier, bureau 450, Sainte-Foy, Québec G1V 2M2 • Tél.: (418) 657-4399 - www.puq.ca

Tiré de: Revue internationale P.M.E., vol. 18, $\mathrm{n}^{\circ}$ 1, sous la direction de Louis Raymond $\cdot$ PME1801N

Tous droits de reproduction, de traduction et d'adaptation réservés 
Même si une transaction visant l'achat de nouveau matériel plus performant est actuellement en cours, la capacité de production actuelle reste faible par rapport au niveau mondial et les potentialités d'exportation de la société sont beaucoup plus importantes ${ }^{10}$, dépassant ainsi la capacité de production annuelle de «A». Aussi, l'outil de production actuel semble vétuste, présentant des signes manifestes de détérioration. La technologie disponible ne permet pas un changement rapide des outils et accessoires de production ${ }^{11}$. Avec sa capacité de production actuelle, la société «A» réalise $60 \%$ de son chiffre d'affaires à l'exportation vers les marchés de la France, la Belgique, les Pays-Bas, l'Allemagne, l'Italie, le Maghreb et quelques pays de l'Afrique de l'Est. Cependant, le diagnostic commercial posé par un cabinet externe $^{12}$ (mai 2000) pointe:

- la rigidité du processus d'acceptation de nouveaux clients, de l'adaptation des conditions commerciales et le manque de flexibilité dans la satisfaction des commandes;

- l'absence d'une fonction marketing et développement de produits;

- la connaissance insuffisante du portefeuille client des représentants étrangers.

En se basant sur l'analyse des documents comptables et financiers de l'entreprise, sur le diagnostic externe élaboré par la banque de la sociétét ${ }^{13}$ (2002) et sur les entretiens semi-directifs que nous avons pu avoir avec le directeur administratif et financier, nous avons pu dégager les problèmes financiers suivants:

- faible valeur ajoutée pour l'activité des tubes induite par l'importance des achats consommés dans le coût global;

- forte exposition aux risques de fluctuation des prix de l'acier en tant que matière première et au risque de change;

- érosion continue des marges, risque financier à cause du poids des charges financières par rapport à l'excédent brut d'exploitation;

- équilibre précaire de la structure financière;

- endettement bancaire à court terme élevé et faible solvabilité.

D'après la direction financière de la société, les causes de ses faiblesses sont aussi liées à la santé du secteur du tube en acier en général, et des réponses d'ordre stratégique doivent être apportées (diversification, abandon de certains produits,

10. Chiffres communiqués par la comptabilité analytique de A.

11. Diagnostic du cabinet ACC en collaboration avec le CETIM France.

12. ACC Consulting.

13. Banque internationale arabe de Tunisie.

Revue internationale P.M.E., vol. 18, n 1, 2005

(C) 2005 - Presses de l'Université du Québec

Édifice Le Delta I, 2875, boul. Laurier, bureau 450, Sainte-Foy, Québec G1V 2M2 - Tél.: (418) 657-4399 - www.puq.ca

Tiré de: Revue internationale P.M.E., vol. 18, $\mathrm{n}^{\circ}$ 1, sous la direction de Louis Raymond $\cdot$ PME1801N

Tous droits de reproduction, de traduction et d'adaptation réservés 
minimisation des risques, etc.). Depuis les années 1990, les dirigeants de «A»se sont rendu compte que le taux de croissance du marché tunisien dans le domaine des tubes en acier est insuffisant pour garantir une croissance acceptable. Cela a poussé ses dirigeants à songer aux marchés extérieurs. Pendant cette période, l'État a mis au point d'importants dispositifs en vue d'accompagner l'exportation tunisienne et le développement international des entreprises nationales. C'est ainsi et à titre non exhaustif que les banques ont commencé à financer sérieusement les exportations et que la douane tunisienne a permis des importations en admission temporaire. Le $\mathrm{CEPEX}^{14}$, qui joue également un rôle très dynamique dans l'accompagnement de l'entreprise exportatrice, accorde une subvention d'État qui rembourse $50 \%$ des coûts de transports (très coûteux pour l'entreprise). Cette subvention, qui représente un déterminant d'importance majeure pour les produits de la société «A», techniquement intéressants mais économiquement à faible valeur ajoutée, doit s'annuler en 2004. Ainsi, les dirigeants de la société «A» se sont trouvés devant l'obligation de chercher de nouveaux créneaux leur permettant la commercialisation des produits sans supporter les coûts de transport relativement importants pour un tel produit de faible valeur ajoutée. Il fallait trouver une solution permettant à «A» d'exporter ses produits avec une valeur ajoutée de production nettement supérieure lui permettant de couvrir ses frais de transport.

\subsection{Le réseau de coopération de A: analyse des apports}

\section{La société $B$}

«B» est une société française de commerce international spécialisée dans l'industrie mécanique. Elle effectue ses achats essentiellement en Afrique du Nord pour vendre en Europe. Depuis le début des années 1990, «A» a fait appel aux services commerciaux de «B». Bénéficiant d'un bon niveau d'intégration sur le marché français grâce à son savoir-faire commercial, cette dernière est devenue le premier partenaire représentant la société en France. «B»se charge de la prospection de marchés, de l'identification des nouvelles opportunités et de la recherche de nouveaux créneaux d'affaires en France et en Europe. Les renseignements fournis permettent de garantir à «A» un nombre minimal d'affaires actuelles et potentielles. L'étape suivante consiste à mettre en contact «A» avec ses clients pour la conclusion des marchés. Le système de rémunération de «B» calculé en pourcentage sur le chiffre d'affaires réalisé en France a garanti à «A» une implication totale de son partenaire français.

14. CEntre de Promotion des Exportations: organisme national d'aide et d'orientation qui vise à promouvoir les exportations et encourager le développement international des entreprises tunisiennes.

Revue internationale P.M.E., vol. $18, \mathrm{n}^{\circ} 1,2005$

(C) 2005 - Presses de l'Université du Québec

Édifice Le Delta I, 2875, boul. Laurier, bureau 450, Sainte-Foy, Québec G1V 2M2 • Tél.: (418) 657-4399 - www.puq.ca

Tiré de: Revue internationale P.M.E., vol. 18, $\mathrm{n}^{\circ}$ 1, sous la direction de Louis Raymond • PME1801N

Tous droits de reproduction, de traduction et d'adaptation réservés 


\section{La société $C$}

PME de nationalité tunisienne, «C $\mathrm{C}$ a comme principal secteur d'activité l'industrie automobile. Certains de ses produits sont à base de tubes. La société représente donc un bon consommateur des produits de «A». Toutefois, les deux sociétés ne se sont pas contentées d'une simple relation client-fournisseur. En se basant sur le savoir-faire technologique de $« \mathrm{C} »$ à façonner les tubes, matière première fournie par «A», les deux sociétés ont instauré un système de co-production de plusieurs produits tubulaires, tels que les produits de collectivités, les glissières de sécurité, les chaises pour les tribunes de stades ou les structures de sièges pour voitures. Pour les dirigeants de $« \mathrm{~A} »$, la relation avec $« \mathrm{C} »$ a permis de construire un banc d'essai de la coopération industrielle et de réduire les risques relatifs de gros investissements en solo, dépassant ainsi les capacités financières de «A». Toutefois, les résultats de la coopération n'ont pas atteint le niveau de satisfaction attendu par les deux sociétés. Si le directeur technique de $« \mathrm{C} »$ a insisté sur le manque de synergie entre les deux sociétés en coopération, le président-directeur général de «A» est plutôt d'avis que l'affaire est une «question de culture» en faisant référence aux entreprises italiennes.

\section{La société $D$}

La société «D» est de nationalité française; elle a été créée en 1985 à la suite de la reprise de certains actifs d'une autre société. Pour les dirigeants la production, la distribution et la location représentent «trois pôles majeurs ${ }^{15}$ » qu'il convenait de repenser. La vocation internationale de la société n'est pas récente. Depuis bon nombre d'années, cette dernière est présente à travers des filiales ou des accords de distribution, en Italie, en Espagne et en Belgique. Actuellement, les ateliers exportent dans 52 pays. Les documents de la production de la société « $\mathrm{D} »$ nous ont permis de dégager trois principaux besoins en matières premières, parmi lesquels nous avons pu détecter les tubes aciers et tôle et les profilés aluminium. Ainsi, «A» se présente à la base comme l'un des fournisseurs de «D». Aussi, «D»a-t-elle signé un accord d'aménagement du travail; ces accords concernent la majeure partie des effectifs et permettent de ramener la durée hebdomadaire de travail à 35 heures. La sauvegarde de la souplesse de fonctionnement indispensable à la satisfaction de la demande du client devrait alors accroître les coûts de la main-d'œuvre de l'entreprise qui représentaient $27 \%$ en 2002 du chiffre d'affaires de l'entreprise. À cet égard, les dirigeants de la société ont déclaré que la délocalisation d'une partie de l'activité de l'entreprise était impérative.

15. Déclarations du président-directeur général de la société.

Revue internationale P.M.E., vol. 18, n 1, 2005

(C) 2005 - Presses de l'Université du Québec

Édifice Le Delta I, 2875, boul. Laurier, bureau 450, Sainte-Foy, Québec G1V 2M2 - Tél.: (418) 657-4399 - www.puq.ca

Tiré de: Revue internationale P.M.E., vol. 18, $\mathrm{n}^{\circ} 1$, sous la direction de Louis Raymond P PME1801N

Tous droits de reproduction, de traduction et d'adaptation réservés 
Les tubes en aciers produits par «A» représentent la matière première la plus importante pour «D». De plus, «A» dispose d'une main-d'œuvre qualifiée et à un coût relativement bas (sept fois plus bas). En revanche, «D», axée essentiellement sur le matériel pour le bâtiment, est l'un des géants mondiaux de la fourniture de matériel pour les bâtiments. Il constitue, en plus, un grand consommateur de tubes en acier (matière première), dispose d'une équipe bien organisée, mais d'une force technologique (R-D) et commerciale trop importantes. Dans ce qui suit, nous présenterons la société «E», fruit de la formation concrète de l'alliance «D-A». Le but étant toujours de chercher les apports pour notre PME.

\section{La société $E$}

La concrétisation juridique de la coopération «A-D» a donné naissance à la société «E», SA de droit tunisien au capital de détenu à $66 \%$ par «D» et $34 \%$ par «A». L'objectif industriel de la nouvelle société vise principalement, dans un premier temps, la fabrication de deux types de produits pour le bâtiment affectés aux marchés italien, espagnol ainsi qu'au marché local. La mise en œuvre de ce programme bénéficie ainsi d'une double impulsion: celle de «D», qui apporte l'outil de travail, le savoir-faire industriel ainsi qu'une force commerciale de dimension internationale; celle aussi de «A» qui, outre la fourniture de tubes, met en œuvre, en collaboration avec « $\mathrm{D} »$, les moyens nécessaires en matière de gestion, d'administration et d'organisation opérationnelle. «A» compte enregistrer une augmentation de $15 \%$ à $20 \%$ de ses produits à l'exportation (achetés par la nouvelle société créée), soit $5 \%$ à $7 \%$ des ventes totales. On estime, à la fin 2003, de 4000 à 4500 tonnes à exporter en plus (une expédition par jour). L'amélioration de la qualité des produits figure aussi parmi les objectifs tracés et visés par cette alliance. Pour ce faire, on envisage de créer, dans une deuxième étape, un bureau d'études tunisien qui doit progressivement intégrer les besoins d'un marché européen compliqué et sophistiqué. La société «A» compte sur son partenaire «D» pour s'occuper de la commercialisation de ses produits en Europe. C'est donc un partenaire qui aura pour tâches principales l'action marketing et le contact client.

\subsection{Schéma de la coopération: interprétation des apports}

Puisque «E» est une société offshore ${ }^{16}$, sa création a permis à «A» de garantir un développement international depuis la Tunisie. En fournissant ses produits à la nouvelle société, «A» continuera à exporter indirectement ses produits de base après l'année 2004, date prévue pour la suspension des subventions accordées à son activité à l'export. Rappelons que ces produits - classés parmi les produits

16. Société exportatrice, fiscalement située en dehors de frontières tunisiennes.

Revue internationale P.M.E., vol. 18, $n^{\circ}$ 1, 2005

(C) 2005 - Presses de l'Université du Québec

Édifice Le Delta I, 2875, boul. Laurier, bureau 450, Sainte-Foy, Québec G1V 2M2 • Tél.: (418) 657-4399 - www.puq.ca

Tiré de: Revue internationale P.M.E., vol. 18, $\mathrm{n}^{\circ} 1$, sous la direction de Louis Raymond $\cdot$ PME1801N

Tous droits de reproduction, de traduction et d'adaptation réservés 
«pauvres» (faible valeur ajoutée) - ne sont pas exportables sans les subventions, car le coût de transport «absorbe» la marge bénéficiaire. Grâce à cette stratégie de coopération tuniso-européenne, les produits de «A» ne seront plus exportés, mais plutôt transformés sous une forme plus complexe (plus de valeur ajoutée). Par ailleurs, sans l'implication combinée des différents partenaires identifiés: «B», « $\mathrm{C} »$ et «D», les produits «A» n'auraient jamais réussi à franchir l'autre côté de la Méditerranée et «E»n'aurait jamais vu le jour. Compte tenu des différentes contraintes, la société «A» $\mathrm{n}$ 'aurait jamais pu convaincre à elle seule ${ }^{17}$ un partenaire de la taille de «D» de s'allier avec elle. Guidé par les résultats que nous avons obtenus, nous envisagions de dégager une représentation du comportement global du réseau constitué par les entreprises en question, ainsi que l'apport des différentes relations sur le développement international de «A». La figure suivante retrace par des flèches les apports des acteurs du réseau à la $\mathrm{PME} \ll \mathrm{A} »$.

Figure 1

Vers une identification plus claire des apports du réseau de coopération de $\mathrm{A}$

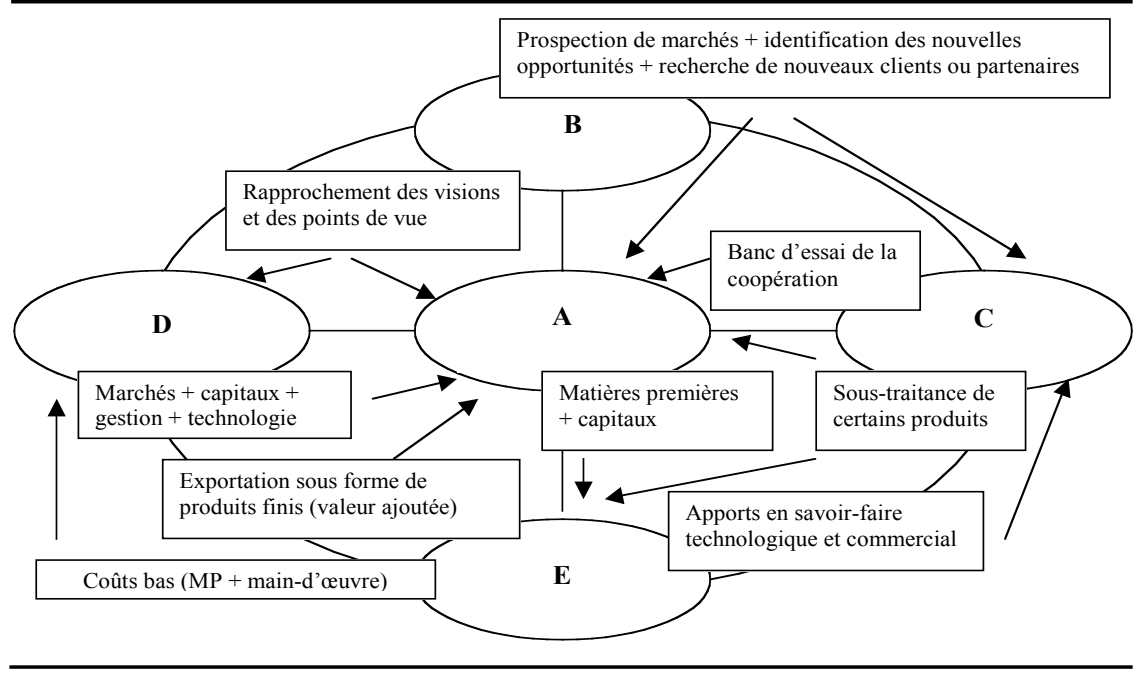

17. Déclaration du président-directeur général de la société « $\mathrm{A} »$.

Revue internationale P.M.E., vol. 18, n 1, 2005

(C) 2005 - Presses de l'Université du Québec

Édifice Le Delta I, 2875, boul. Laurier, bureau 450, Sainte-Foy, Québec G1V 2M2 • Tél.: (418) 657-4399 - www.puq.ca

Tiré de: Revue internationale P.M.E., vol. 18, $\mathrm{n}^{\circ} 1$, sous la direction de Louis Raymond $\cdot$ PME1801N

Tous droits de reproduction, de traduction et d'adaptation réservés 


\section{Vers une approche mieux adaptée...}

\subsection{Les enseignements théoriques du cas}

Nous allons nous intéresser aux apports spécifiques des relations partenariales observées autour de «A». Une confrontation de ces apports aux faiblesses de cette dernière dans un stade ultérieur guidera nos propos.

À la différence de plusieurs partenaires commerciaux de la société «A» en Europe, la tâche de « $\mathrm{B}$ » dépasse la simple prospection et commercialisation de produits vers de nouveaux clients généralement inconnus par la société tunisienne et qui peut atteindre parfois $15 \%$ du chiffre d'affaires de «A» à l'exportation. Ainsi, au-delà du savoir-faire et du rayonnement commercial, la relation «A - B » constitue un canal d'information pour la PME tunisienne. Après une première phase d'identification et de connaissance, une relation plus étroite sur le marché avec les partenaires potentiels d'affaires est établie. À ce stade, la contribution de «B» est primordiale. Connaissant généralement les parties en contact, cette dernière cherche à établir une relation plus durable entre elles, puisque son intérêt est aussi en jeu. D'ailleurs, plusieurs parties ont jugé le rôle de la société «B» comme «moteur» garantissant le bon fonctionnement et la continuité du réseau de «A»: c'est une «coopération pour la coopération ${ }^{18}$ ». L'effort déployé par «B», notamment au regard de la coordination et de la recherche des synergies, permet donc d'aider «A» et de compléter ses spécificités dans la conviction de nouveau partenaire par un processus évolutif.

Disposant d'une usine à proximité, la société « $\mathrm{C}$ » représente l'un des premiers partenaires industriels de «A». Le domaine d'activités de «C», basé essentiellement sur des produits de «A», ainsi que son savoir-faire technologique ont constitué un véritable stimulant pour la coopération. En effet, les moyens financiers de «A» ne lui permettent pas d'opter pour une intégration verticale en internalisant une partie supplémentaire des activités tubulaires, car une telle démarche requiert des investissements importants. De plus, en tenant compte du contexte actuel et des spécificités de «A», le risque lié à la création d'une nouvelle activité n'est pas négligeable. La coopération qui a pris naissance entre entreprises tunisiennes se présente donc comme opportune mais surtout à moindres coûts, dans la mesure où une telle coopération permet à «A» d'atteindre une certaine «masse» sur le marché mondial.

À travers sa coopération avec «A», «D» cherchait principalement à diminuer le prix de revient de ses produits à base de tubes d'acier. En effet, en s'approvisionnant en Tunisie, par l'intermédiaire de «E», qui assurerait par ses moyens humains,

18. Selon le directeur commercial de « $\mathrm{A} »$.

Revue internationale P.M.E., vol. 18, $\mathrm{n}^{\circ} 1,2005$

(C) 2005 - Presses de l'Université du Québec

Édifice Le Delta I, 2875, boul. Laurier, bureau 450, Sainte-Foy, Québec G1V 2M2 • Tél.: (418) 657-4399 - www.puq.ca

Tiré de: Revue internationale P.M.E., vol. 18, $\mathrm{n}^{\circ} 1$, sous la direction de Louis Raymond $\cdot$ PME1801N

Tous droits de reproduction, de traduction et d'adaptation réservés 
financiers et techniques disponibles sur place la fabrication d'une partie de ces produits, «D» a pu réaliser des économies tant sur les coûts d'approvisionnement, par l'élimination des coûts de transport maritime, que sur les coûts salariaux, la main-d'œuvre dans ce secteur demandant des salaires beaucoup plus bas en Tunisie qu'en France. Par ailleurs, la relation «A - D» apparaît comme l'une des formes les plus poussées de la coopération contractuelle. En concluant une telle alliance, les deux sociétés ont opté pour une addition complémentaire de compétences. Le but de «A» était d'élaborer des solutions et systèmes plus complexes, par la contribution financière, technologique, commerciale et managériale de « $\mathrm{D} »$. À cet égard, la contribution de $« \mathrm{~B} »$ au bon fonctionnement du système s'est révélée très importante, puisque cette dernière se présente comme un troisième partenaire coordinateur possédant un savoir-faire commercial. De plus, le poids de $« \mathrm{C} »$ comme partenaire industriel de «A» a permis à cette dernière de bien négocier sa coopération avec «D». Ainsi, «E» peut être considérée comme une forme hybride de développement international grâce à laquelle « $\mathrm{A} »$ a pu garantir un marché durable représentant au moins $20 \%$ des exportations totales de l'entreprise. Nous pouvons donc affirmer que la diversité des apports des différentes sociétés autour de «A» a constitué un atout incontestable pour le développement de cette dernière malgré ses contraintes spécifiques. En effet, les apports commerciaux de «B» et de «D» ont facilité l'accès de «A» sur la scène internationale. Le matériel et le savoir-faire technologique de «C» ont permis à cette société («A») d'atteindre «une masse» plus convaincante, tandis que la conclusion d'une alliance avec «D» lui a permis de complexifier son activité à moindres coûts et risques, et ce, en mobilisant des compétences externes.

Partant de l'idée de l'importance de la coopération pour le développement international des entreprises, nous avons mis l'accent sur les avantages spécifiques au réseau comme modalité de croissance à part entière. Par ailleurs, pour mettre en évidence l'utilité de notre proposition, nous nous sommes basé empiriquement sur l'étude de cas. Nous avons pu dégager les apports des différents partenaires de «A» que nous avons confrontés à ses faiblesses. À travers cette confrontation, nous pouvons dire que les propos avancés sur l'apport des réseaux de coopération sont vérifiés.

En reprenant les avantages spécifiques proposés par Dunning (1993), nous avons pu associer les avantages de la coopération en réseau dégagés par la présente étude empirique. Nous rappelons que notre objectif de départ était de justifier la complémentarité et parfois la substitution de ce type d'avantages aux avantages spécifiques. Cependant, il est à noter que les avantages coopératifs dégagés ne sont pas exclusifs, puisqu'ils sont propres à notre cas. Cette confrontation vise à alimenter notre réflexion sur une conception adaptée aux PME.

Revue internationale P.M.E., vol. 18, n 1, 2005

(C) 2005 - Presses de l'Université du Québec

Édifice Le Delta I, 2875, boul. Laurier, bureau 450, Sainte-Foy, Québec G1V 2M2 - Tél.: (418) 657-4399 - www.puq.ca

Tiré de: Revue internationale P.M.E., vol. 18, $\mathrm{n}^{\circ} 1$, sous la direction de Louis Raymond PME1801N

Tous droits de reproduction, de traduction et d'adaptation réservés 
Tableau 3

\section{Confrontation des avantages spécifiques à l'entreprise aux avantages spécifiques au réseau}

\begin{tabular}{ll}
\hline $\begin{array}{l}\text { Avantages spécifiques à l'entreprise } \\
\text { (Dunning, 1988, 1993) }\end{array}$ & $\begin{array}{l}\text { Avantages spécifiques au réseau } \\
\text { (prolongement des réflexions de }\end{array}$ \\
\hline$\checkmark$ Gunning, 1995, 2001)
\end{tabular}

Dans la discussion théorique qui suit, une réflexion sur un cadre-synthèse adapté aux PME est proposée. Notre but n'est pas de présenter un modèle généralisable et notre approche se veut résolument exploratoire, et ce, pour deux raisons. D'abord, parce que la complexité déjà relevée dans l'identification empêche de tenir compte de tous les cas de figures possibles; ensuite, parce que la logique de notre démarche est inspirée de cas particuliers d'entreprises.

\subsection{Essai de discussion théorique}

La première conception de Dunning $(1988,1993)$ défend la nature idiosyncrasique des ressources et compétences censées être maîtrisées par l'entreprise. En effet, l'approche par les ressources de base est individualiste et se focalise essentiellement sur les spécificités de la firme, en délaissant la coordination interentreprises. Cependant, le réseau semble parfaitement compatible avec un environnement 
internationalisé qui impose de connecter les moyens et les compétences des unités délocalisées. De même, il semble que ce type d'action apparaisse surtout dans des secteurs fragmentés, favorables à une dissémination des tâches et des responsabilités comme les services, le textile, la chaussure ou l'édition.

Pour les PME, la modalité de présence à l'étranger la plus souvent utilisée est l'exportation directe, indirecte ou concertée. De plus, les nouvelles technologies offrent aux entreprises de très bonnes opportunités à l'international. Ainsi, l'investissement dans des implantations étrangères n'est souvent pas une modalité préférée par les PME, surtout avec les contraintes que comportent ces dernières et que nous avons déjà évoquées. Dans la formulation du modèle global, le paradigme éclectique se base sur trois types d'avantages favorisant l'internationalisation: les avantages spécifiques à l'entreprise, les avantages de localisation géographique et les avantages de l'internalisation des activités. Quelle que soit la modalité de présence à l'étranger, la conception défend le déterminisme des avantages spécifiques pour l'entreprise. Si ce modèle peut être valable pour certaines PME, il ne l'est que pour une minorité. En effet, les caractéristiques d'une PME sont loin de lui offrir des avantages spécifiques (taille, capacité financière, grande technologie, etc.) pour l'internalisation. De plus, si nous nous référons aux fondements de base de l'approche réseau comme forme intermédiaire entre le marché et la hiérarchie (Williamson, 1975; Thorelli, 1986), nous pouvons avancer que le recours des PME à la coopération se présente comme «la» solution qui permet de dépasser les faiblesses liées à la taille de l'entreprise, mais aussi d'éviter les carences du marché. C'est à partir de cette logique que nous allons proposer les avantages spécifiques aux réseaux, résultat de l'aide extérieure apportée à l'entreprise par ses relations de coopération externe, comme avantages complémentaires aux caractéristiques spécifiques de la PME. Outre les voies de l'internalisation ou de l'externalisation, une troisième voie flexible et surtout adaptée s'ouvre aux entreprises: le réseau caractérisé par le maintien de l'indépendance capitalistique et juridique de chaque partenaire, conjugué à une forte volonté de collaborer et de mobiliser les compétences respectives au sein d'une même chaîne de talents et de savoir-faire, s'inscrit dans un ensemble d'échanges avec les autres (Balantzian, 1997), sans pour cela perdre son identité et son autonomie.

Toutefois, la question qui se pose ici est de savoir si le réseau est une ressource en soi ou si c'est une porte d'accès aux (ou encore une source de) ressources? En effet, si l'on admet que la capacité à coordonner et à faire coopérer plusieurs ressources constitue une compétence, les compétences relationnelles d'une entreprise représentent une force intangible et quelquefois durable, qui permet à cette dernière d'obtenir des conditions plus satisfaisantes que celles des concurrents qui traitent avec les mêmes acteurs. La relation de réseau peut être considérée comme un actif intangible et, donc, comme une ressource spécifique au sens de Wernerfelt (1984). D'ailleurs, c'est sur cette même logique que se sont basés les travaux de Dunning (1995) sur la reconfiguration du paradigme éclectique.

Revue internationale P.M.E., vol. 18, nº 1, 2005

(C) 2005 - Presses de l'Université du Québec

Édifice Le Delta I, 2875, boul. Laurier, bureau 450, Sainte-Foy, Québec G1V 2M2 - Tél.: (418) 657-4399 - www.puq.ca

Tiré de: Revue internationale P.M.E., vol. 18, $\mathrm{n}^{\circ} 1$, sous la direction de Louis Raymond • PME1801N

Tous droits de reproduction, de traduction et d'adaptation réservés 
D'un autre côté, l'action en réseau permet de mobiliser des ressources que l'on ne possède pas forcément, mais qui peuvent être disponibles chez les partenaires en relation. Dans cette optique, Marchesnay (2002) parle du «rôle des réseaux, comme facteur d'échange et d'appropriation des ressources». À cet égard, Dunning (2001) propose une «extension naturelle» de ses réflexions précédentes (1988, 1993, 1995) par ce qu'il appelle «the relational-assets». Par conséquent, une réflexion sur des modifications à apporter au modèle OLI est proposée. Pour les PME qui ne disposent pas d'avantages spécifiques ou qui en manquent, nous proposons les avantages spécifiques au réseau comme substitut ou complément. En effet, la recherche d'avantages par la coopération en réseau offre des opportunités pour l'internationalisation des PME, souvent pauvres en avantages spécifiques. Si les propositions de Dunning (1995) intègrent ce type d'avantages dans la dimension des avantages spécifiques de l'entreprise, il nous a semblé pertinent de les proposer comme dimension à part entière favorisant l'internationalisation des PME. La nature externe et dynamique des réseaux, d'une part, la place de ces dernières comme formule intermédiaire, d'autre part, et les travaux de Heitz (2000) sur la spécificité des actifs réseaux ont conforté notre choix.

Tableau 4

Vers une nouvelle logique adaptée du modèle OLI

\begin{tabular}{ll}
\hline $\begin{array}{l}\text { Logique du modèle OLI } \\
\text { (Dunning, 1993, 1995) }\end{array}$ & Logique de notre proposition: OLN* \\
\hline Avantages spécifiques. & Avantages spécifiques et/ou avantages réseau. \\
Avantages d'internalisation. & $\begin{array}{l}\text { Avantages réseau. } \\
\text { Se spécialiser dans (et donc internaliser) les activités } \\
\text { où l'on possède un avantage concurrentiel et agir en } \\
\text { réseau pour les activités où l'on est moins bon. }\end{array}$ \\
\hline
\end{tabular}

Avantages de localisation. Avantages de localisation.

* OLN: Ownership-specific advantages, Localisation advantages and Network-related advantages.

Par ailleurs, à partir des enseignements du cas, nous nous sommes rendu compte que notre proposition théorique de départ ne prend pas en considération l'intervention de l'État tunisien matérialisée par la subvention du CEPEX. Même si elle avait un caractère provisoire, cette subvention était d'une importance majeure pour la première phase du développement international de «A». De plus, la Tunisie, comme pays d'origine de l'entreprise, présente plusieurs avantages tels que les faibles coûts de la main-d'œuvre, la réglementation fiscale et la proximité

Revue internationale P.M.E., vol. $18, \mathrm{n}^{\circ} 1,2005$

(C) 2005 - Presses de l'Université du Québec

Édifice Le Delta I, 2875, boul. Laurier, bureau 450, Sainte-Foy, Québec G1V 2M2 • Tél.: (418) 657-4399 - www.puq.ca

Tiré de: Revue internationale P.M.E., vol. 18, $\mathrm{n}^{\circ} 1$, sous la direction de Louis Raymond PME1801N

Tous droits de reproduction, de traduction et d'adaptation réservés 
culturelle et géographique de l'Europe. Ces avantages peuvent représenter un atout incontestable à l'internationalisation des PME tunisiennes. Si les propositions de Dunning (1993) prévoyaient l'intégration de ce type d'avantages dans la dimension des avantages spécifiques de l'entreprise, il nous a semblé pertinent de placer le cadre OLN proposé dans le contexte dans lequel agit la PME. Ce qui converge avec les travaux de Håkansson (1982) et permet de tenir compte de l'impact des avantages offerts par l'environnement de l'entreprise (subventions, aides directes de l'État, programmes de mise à niveau, conditions initiales favorables, etc.). Par conséquent, le modèle d'aboutissement suivant nous semble mieux adapté pour appréhender l'internationalisation des PME:

- bien qu'ils semblent mieux adaptés à l'implantation physique et aux entreprises de grande taille, les avantages spécifiques à l'entreprise prévus dans le modèle de base sont gardés et complétés par la dimension «avantages liés aux réseaux»;

- tenant compte des propos avancés sur les avantages coopératifs (par les réseaux) et la non-exclusivité de l'internalisation comme chemin pour le développement international de la PME, nous proposons les avantages liés au réseau comme troisième voie intermédiaire, plus accessible aux PME et donc comme substitut aux avantages de l'internalisation;

- nous gardons les avantages de localisation comme dimension à part entière du modèle, compte tenu de l'intérêt qu'elle porte aux spécificités du pays où la PME compte se développer à l'international;

- en nous basant sur les enseignements empiriques de notre cas, nous plaçons notre modèle dans un cadre «avantages liés au contexte». Ces derniers, prenant en compte les avantages offerts par l'environnement de l'entreprise, interviennent comme champ dans lequel s'articulent les trois types d'avantages précités.

Le cadre théorique proposé permet d'appréhender plus finement le développement international des PME. Notons toutefois qu'il n'est pas nécessaire pour une PME de combiner tous les avantages à la fois, car il s'agit simplement d'avantages complémentaires, dont l'articulation dans un contexte favorable rendra l'internationalisation plus facile.

\section{Conclusion}

La reprise du modèle OLI du paradigme éclectique de Dunning en utilisant une approche par les réseaux permet de dépasser les limites des principaux courants théoriques antérieurs sur l'internationalisation des PME, pour offrir une démarche appropriée et adaptée à leurs spécificités. Si les principaux travaux sur le sujet

Revue internationale P.M.E., vol. 18, $\mathrm{n}^{\circ} 1,2005$

(C) 2005 - Presses de l'Université du Québec

Édifice Le Delta I, 2875, boul. Laurier, bureau 450, Sainte-Foy, Québec G1V 2M2 • Tél.: (418) 657-4399 - www.puq.ca

Tiré de: Revue internationale P.M.E., vol. 18, $\mathrm{n}^{\circ} 1$, sous la direction de Louis Raymond • PME1801N

Tous droits de reproduction, de traduction et d'adaptation réservés 
insistent généralement sur l'importance du facteur humain, le nouveau cadre de réflexion théorique proposé à l'issue de la phase empirique vise à prendre en considération les différents déterminants favorisant l'internationalisation des entreprises de petite et moyenne taille. En mettant l'accent sur les avantages coopératifs des réseaux, nous avons pu aboutir à un modèle plus intégratif. Toutefois, les difficultés théoriques liées à la PME expliquent notre recours au modèle du paradigme éclectique moins spécialisé. Malgré les risques et limites d'une telle démarche pour la définition des apports, l'approche proposée fournit les éléments d'une bonne argumentation pour sensibiliser les dirigeants de PME sur les apports des réseaux d'entreprises à l'international.

\section{Bibliographie}

Aaby, N.E et S.F. SLAter (1989), «Management influences on export performance: a review of the empirical literature 1978-1988», International Marketing Review, vol. $6, \mathrm{n}^{\circ} 4$, p. 7-26.

Aharoni, Y. (1996), «Ownerships, networks and coalitions: the globalisation of professional business services», dans Y. Aharoni, Coalitions and Competition, Londres, Routledge.

Balantzian, G. (1997), L'avantage coopératif: le partenariat, la coopération, l'alliance stratégique, Paris, Éditions d'Organisation.

Bilkey, W. et G. Tesar (1977), «The export behavior of small-sized Wisconsin manufacturing firms », Journal of International Business Studies, vol. 8, n 1, p. 93-98.

Buörkman, I. (1990), On Economic and Decision Process Oriented Perspectives to Analyzing Foreign Direct Investment Decisions, Document de travail 202, Swedish School of Economics and Business Administration, Helsinki.

Bloodgood, J.M., H.J. SapienZa et J.G. Almeida (1996), «The internationalization of new high potentiel US ventures: antecedents and outcomes», Entrepreneurship, Theory and Practice, vol. 20, n ${ }^{\circ}$ 4, p. 61-76.

Brousseau, E. (1996), «Théorie des contrats, coordination interentreprises et frontière de la firme», Documents de recherche ATOM, $\mathrm{n}^{\circ}$ 1996-03.

BucKLEy, P.J. et M. CAsson (1976), The Future of the Multinational Entreprise, Londres, The Macmillan Press.

BYÉ, M. (1987), Relations économiques internationales, Paris, Dalloz.

Caves, R.E. (1982), Multinational Enterprise and Economic Analysis, Cambridge, Mass., Cambridge University Press.

CAvusgiL, S.T. (1976), «Organisation determinants of firm's behaviour: an empirical analysis», Thèse de doctorat non publiée, citée dans M. Ortiz-Buonafina (1990), «Small business exporting», Journal of Global Marketing, vol. 3-4, no 36, p. 89-102.

Revue internationale P.M.E., vol. $18, \mathrm{n}^{\circ} 1,2005$

(C) 2005 - Presses de l'Université du Québec

Édifice Le Delta I, 2875, boul. Laurier, bureau 450, Sainte-Foy, Québec G1V 2M2 - Tél.: (418) 657-4399 - www.puq.ca

Tiré de: Revue internationale P.M.E., vol. 18, $\mathrm{n}^{\circ}$ 1, sous la direction de Louis Raymond • PME1801N

Tous droits de reproduction, de traduction et d'adaptation réservés 
Cavusgil, S.T. et J.R. Nevin J.R. (1981), «Internal determinants of export marketing behavior: an empirical investigation», Journal of Marketing Research, vol. 18, $\mathrm{n}^{\circ} 1$, p. $114-119$.

Chipman, J.S. (1965), «A survey of the theory of international trade: part I, the classical theory», Econometrica, ${ }^{\circ} 33$, p. 477-519.

CoASE, R.H. (1937), «The nature of the firm», Economica, vol. 4, n 16, p. 386-405, traduction française «La nature de la firme», Revue française d'économie, vol. 2, $\mathrm{n}^{\circ} 1,1987$, p. 133-156.

Coeurderoy, R. et M. Ingham (2004), «Le concept de réseau en management stratégique: de l'exclusion à la centralité», dans Les réseaux: dimensions stratégiques et organisationnelles, Paris, Economica.

Coriat, B. et O. Weinstein (1995), Les nouvelles théories de l'entreprise, Paris, Librairie générale française, coll. «Le livre de poche», $218 \mathrm{p}$.

Cyert, R.M. et J.G. March, (1964), «A behavioural theory of the firm», The American Economic Review, vol. 54, n 2, partie 1, p. 144-148.

Defeuilley, E. et M. Vahabi (1996), «Transaction cost theory and evolutionary economics, the questionable synthesis», Colloque à la Sorbonne, Paris, 19-20 septembre.

DE LA VILle, I. (2000), «La recherche idiographique en management stratégique: une pratique en quête de méthode?», Revue Finance, Contrôle, Stratégie, septembre, p. 73-99.

De Toni, A. et G. NAssimbeni (2001), «The export propensity of small firms: a comparison of organisational and operational management levers in exporting and non-exporting units», International Journal of Entrepreneurial Behaviour \& Research, vol. 7, $\mathrm{n}^{\circ} 4$, p. 132-147.

DunNing, J.H. (1977), Trade Location of Economic Activity and Multinational Entreprise: The International Location of Economic Activity, Londres, Macmillan, p. 395-418.

DunNING, J.H. (1988), «The eclectic paradigm of international production: a restatement and some possible extensions», Journal of International Business Studies, vol. 19, $\mathrm{n}^{\circ} 1$, p. $1-31$.

DunNing, J.H. (1993), Multinational Entreprises and the Global Economy, Reading, Mass., Addison-Wesley.

DunNING, J.H. (1995), «Reappraising the eclectic paradigm in age of alliance capitalism», Journal of International Business Studies, vol. 26, p. 461-491.

DuNNING, J.H. (2001), «The eclectic paradigm of international production: past, present and future», International Journal of the Economics of Business, vol. 8, nº 2, p. 173-190.

DuNNING, J.H. (2002), «Perspectives on international business research: a professional autobiography fifty years researching and teaching international business », Journal of International Business Studies, vol. 33, n 4, p. 817-838.

EMERSON, R.M. (1962), «Power-dependence relations», American Sociological Review, vol. 17 , p. $31-41$.

Revue internationale P.M.E., vol. 18, nº 1, 2005

(C) 2005 - Presses de l'Université du Québec

Édifice Le Delta I, 2875, boul. Laurier, bureau 450, Sainte-Foy, Québec G1V 2M2 • Tél.: (418) 657-4399 - www.puq.ca

Tiré de: Revue internationale P.M.E., vol. 18, no 1, sous la direction de Louis Raymond PME1801N

Tous droits de reproduction, de traduction et d'adaptation réservés 
FErguson, W.-D. (1996), «Explaining the rising wage-productivity gap of the 1980s: Effects of declining employment and unionisation », Review of Radical Political Economics, vol. $28, \mathrm{n}^{\circ} 2$, p. $77-115$.

Granovetter, M. (1985), «Economic action and social structure», American Journal of Sociology, ${ }^{\circ}$ 91, p. 481-551.

Gulati, R., N. Nohria et A. Zaheer (2000), «Strategic networks», Strategic Management Journal, vol. 21, p. 203-215.

HÅKANsSON, H. (dir.) (1982), International Marketing and Purchasing of Industrial Goods: An Interaction Approach, Chichester, Wiley, $556 \mathrm{p}$.

HÅKANSSON, H. et J. JoHANSON (1993), «The network as a governance structure. Interfirm cooperation beyond markets and hierarchies», dans G. Grabher, The Embedded Firm. On the Socioeconomics of Industrial Networks, Londres, Routledge, p. 35-51.

HecKSCHER, E.F. (1949), «The effect of foreign trade on distribution of income», dans H.E. Ellis et L.A. Metzler (dir.), The Theory of International Trade, Philadelphie, The Blakiston Company, p. 272-300.

Heitz, M. (2000), «Les coopérations interentreprises: présentation d'une grille de lecture», Revue Finance, Contrôle, Stratégie, vol. 3, n 4, p. 57-81.

Hirsch, F. (1976), Social Limits to Growth, Cambridge, Harvard University Press.

Huault, I. (1998), Le management international, Paris, La Découverte.

Hymer, S.H. (1960), The International Operations of National Firms: A Study of Foreign Direct Investment, Cambridge, Mass., MIT Press.

Hymer, S.H. (1968), «La grande "corporation" multinationale: analyse de certaines raisons qui poussent à l'intégration internationale des affaires», Revue économique, $\mathrm{n}^{\circ} 6$, p. 464-481.

JACQUemot, P. (1990), La firme multinationale: une introduction économique, Paris, Economica.

JARILlo, J.C. (1988), «On strategic network», Strategic Management Journal, vol. 9, p. $31-41$.

JARILLO, J.C. (1990), «Comment on transaction costs and networks», Strategic Management Journal, vol. 11, p. 497-499.

Johanson, J. et L.G. MatTson (1987), «Interorganizational relations in industrial systems: a network approach compared with transaction coast approach», International Studies of Management and Organization, vol. 17, $\mathrm{n}^{\circ}$ 1, p. 34-48.

JOHANSON, J. et J.E. VAHLNE (1977), «The internationalization process of the firm: a model of knowledge development on increasing foreign commitment», Journal of International Business Studies, p. 220-234.

JohANSON, J. et J.E. VAHLNE (1993), «The internationalization process of the firm: a model knowledge development and increasing foreign market commitments», dans P.J. Buckley et P. Ghauri (dir.), The Internationalization of the Firm: A Reader, Londres, Academic Press.

Revue internationale P.M.E., vol. $18, \mathrm{n}^{\circ} 1,2005$

(C) 2005 - Presses de l'Université du Québec

Édifice Le Delta I, 2875, boul. Laurier, bureau 450, Sainte-Foy, Québec G1V 2M2 - Tél.: (418) 657-4399 - www.puq.ca

Tiré de: Revue internationale P.M.E., vol. 18, $\mathrm{n}^{\circ}$ 1, sous la direction de Louis Raymond • PME1801N

Tous droits de reproduction, de traduction et d'adaptation réservés 
Johanson, J. et F.P. Wiedersheim (1975), «The internationalization of the firm: four Swedish cases», The Journal of Management Studies, p. 305-322.

Julien, P.-A. (dir.) (1994), Les PME: bilan et perspectives, Paris, Economica.

Julien, P.-A. (1997), «Pour une définition des PME», dans P.-A. Julien (dir.), Les PME: bilan et perspectives, Paris, Economica.

JuliEn, P.-A. (dir.) (2002), L'importance des PME à forte croissance dans l'économie, Sainte-Foy, Presses de l'Université du Québec.

KAYNAK, E. (1992), «A cross regional comparison of export performance of firms in two Canadian regions », Management International Review, vol. 32, n 2, p. 163-180.

Kindleberger, C.P. (1969), American Business Aboard, New Haven, Yale University Press.

Kogut, B. (1985), «Designing global strategies: comparative value added-chains», Sloan Management Review, p. 15-27.

LECOQ, X. (2003), Comportements d'acteurs et dynamique d'un réseau interorganisationnel: le phénomène des écarts relationnels, Thèse de doctorat soutenue le 25 novembre, IAE de Lille.

LEONIDOU, L.C. (1997), «Finding the right information mix for the export manager», Long Range Planning, vol. 30, $\mathrm{n}^{\circ}$ 4, p. 572-584.

LEONIDOU, L.C. et C.S. KATSIKEAS (1996), «The export development process: an integrative review of empirical models», Journal of International Business Studies, vol. 27, nº 3.

Manolova, T.S., C.G. Brush, L.F. Edelman et P.G. Greene (2002), «Internationalization of small firms. Personal factor revisited», International Small Business Jounal, vol. $20, \mathrm{n}^{\circ} 1$, p. $9-31$.

March, J.G. et H.A. Simon (1958), Organizations, New York, Wiley.

March, J.G. et H.A. Simon (1969), Les organisations, Paris, Dunod.

Marchesnay, M. (1991), «La P.M.E.: une gestion spécifique», Économie rurale, n 206 , p. 11-17.

MaRChESNAY, M. (2002), Pour une approche entrepreneuriale de la dynamique ressourcescompétences, Les Éditions de l'ADREG, site Web <asso.nordnet.fr/adreg>

Mundell, R.A. (1957), «Transport costs in international trade theory», Canadian JE.

Nohria, N. et R.G. Eccles (1992), Network and Organizations: Structure, Form, and Action, Boston, Mass., Harvard Business School Press.

Ohlin, B. et E.F. Heckscher (1933), Interregional and International Trade, Cambridge, Harvard University Press.

Phatak, A.-V. (1992), International Dimensions of Management, $3^{\mathrm{e}}$ édition, Boston, PWS-Kent

Piore, M.J. et C.F. Sabel (1984), The Second Industrial Divide Possibilities for Prosperity, New York, Basic Books.

Porter, M. (1986), Competition in Global Industries, Boston, Harvard Business School Press.

Revue internationale P.M.E., vol. 18, $\mathrm{n}^{\circ} 1,2005$

(C) 2005 - Presses de l'Université du Québec

Édifice Le Delta I, 2875, boul. Laurier, bureau 450, Sainte-Foy, Québec G1V 2M2 - Tél.: (418) 657-4399 - www.puq.ca

Tiré de: Revue internationale P.M.E., vol. 18, $\mathrm{n}^{\circ} 1$, sous la direction de Louis Raymond • PME1801N

Tous droits de reproduction, de traduction et d'adaptation réservés 
Porter, M. (1991), «The competitive advantage of nations, Michael E. Porter on competition and strategy», Harvard Business Review, Paperback n 90079.

Powell, W. (1990), «Neither market nor hierarchies: networks forms of organization», Research in Organizational Behavior, vol. 12, p. 295-336.

REID, S. (1984), «Information acquisition and export entry decisions in small firms », Journal of Business Research, vol. 12, n 2, p. 141-157.

RicARDO, D. (1817), On the principles of political economy and taxation, Londres, John Munay.

Rugman, A.-M. et E. Lorraine (1985), Multinationals and Transfer Pricing, Londres, Croom Helm; New York, St. Martin's Press, 336 p.

SAmuelson, P.A. (1948), «International trade and the equalisation of factor prices», Economic Journal.

Simon, H. (1957), Administrative Behavior, $2^{\mathrm{e}}$ édition, New York, The Free Press.

Sмiтh, A. (1776), Recherche sur la nature et les causes de la richesse des nations, édition traduite en 1949.

Soerensen, O. et A. NedergaArd (1993), «Management decision making in an international context: the case for intuition and action learning. International business and Europe after 1992», dans V. Simoes, Les Actes de la XIX ${ }^{e}$ Conférence annuelle EIBA, p. 345-361, Lisboa, décembre.

Thorelli, H.B. (1986), «Networks: between markets and hierarchies», Strategic Management Journal, vol. 7, p. 37-51.

TORRÈs, O. (dir.) (1998), PME: de nouvelles approches, Paris, Economica, coll. «Recherche en gestion», $187 \mathrm{p}$.

Torrès, O. (1999), Les PME, Paris, Flammarion, coll. «Dominos», 128 p.

UzzI, B. (1997), «Social structure and competition in interfirm networks: the paradox of embeddedness », Administrative Science Quarterly, vol. 42, mars, p. 35-67.

VERNON, R. (1966), «International investment and international trade in the product cycle», Quarterly Journal of Economy, mai, p. 190-207.

WeLCH, L. et R. LUOSTARINEN (1988), «Internationalization evolution of a concept», Journal of General Management, vol. 14, n 2, p. 34-55.

Wernerfelt, B. (1984), «A resource-based view of the firm», Strategic Management Journal, vol. 5, p. 171-180.

Williamson, O.E. (1975), Market and Hierarchies: Analysis and Antitrust Implication, New York, The Free Press.

YIn, R. (1990), Case Studies Research: Design and Methods, Applied Social Research Series, Newbury Park, Sage, vol. 2.

Revue internationale P.M.E., vol. $18, \mathrm{n}^{\circ} 1,2005$

(C) 2005 - Presses de l'Université du Québec

Édifice Le Delta I, 2875, boul. Laurier, bureau 450, Sainte-Foy, Québec G1V 2M2 • Tél.: (418) 657-4399 - www.puq.ca

Tiré de: Revue internationale P.M.E., vol. 18, $\mathrm{n}^{\circ} 1$, sous la direction de Louis Raymond • PME1801N

Tous droits de reproduction, de traduction et d'adaptation réservés 